\title{
ULTRASTRUCTURAL VARIABILITY OF MACROPHAGES IN THE WALL OF SELECTED AORTO-CORONARY BYPASS GRAFTS
}

\author{
Bartłomiej Perek ${ }^{1}$, Katarzyna Kowalska², Agnieszka Malińska², Marek Jemielity ${ }^{1}$, Michał Nowicki ${ }^{2}$
}

\begin{abstract}
Macrophages, detected as CD68+ cells, are considered to have marked contribution to aorto-coronary grafts disease. The purpose of this study was to find any ultrastructural differences in CD68+ cells between arterial and venous aorto-coronary grafts.

The surplus segments of radial artery (RA) and saphenous vein (SV) were obtained from 50 patients with the mean age of $63.4 \pm 9.2$ years who undergo elective coronary artery bypass grafting (CABG). The vascular segments were analyzed by means of both light (to assess number and distribution of macrophages within their walls) and transmission electron microscopy (to evaluate ultrastructure of CD68+ cells in the vessel layers).

Histological analysis revealed that not only more macrophages (median (25th; 75th percentile)) were found on the transverse sections of veins $(95(67 ; 135))$ than arteries $(66(43 ; 108))(p<0.05)$ but also at least of $50 \%$ of them were found in the tunica intima and tunica media in SV while only $30 \%$ in RA. TEM studies showed that biological activity of macrophages depended on CD68+ location and was irrespective of the vessel type. Those found in the tunica intima and tunica media presented ultrastructure typical for active cells rich in numerous lysosomes, well developed rough endoplasmic reticulum and Golgi apparatus whereas adventitial macrophages for unreactive residual cells.

Ultrastructural characteristics of both forms of macrophages infiltrating wall of aorto-coronary grafts is similar irrespective of the vessel type. More active cells in the inner layers of the venous conduits may contribute to their inferior outcomes compared to the arteries.
\end{abstract}

Running title: Macrophages and aorto-coronary grafts

Keywords: coronary artery bypass graft, aorto-coronary graft failure, macrophage, radial artery, saphenous vein 


\section{Introduction}

Coronary artery bypass grafting (CABG), in spite of enormous progress in interventional cardiology, is still a procedure of choice in treatment of multi-vessel coronary artery disease (MVD) with diffuse atherosclerotic plaques [1]. It must be stressed that the optimal choice of aorto-coronary grafts to achieve permanent good outcomes is as important as technical surgical skills to perform successful CABG [2]. An importance of complete revascularization, defined as grafting all severely stenotic and occluded arteries, with respect to clinical improvement of coronary artery disease (CAD) was supported in the previous reports [3]. Moreover, occlusion of aorto-coronary bypasses makes cardiologists and/or cardiac surgeons to perform repeat and less effective revascularization procedures [4].

Currently, three types of vessels are routinely employed in CABG procedures: internal thoracic (ITA), radial arteries (RA) and saphenous veins (SV). Undoubtedly, the best vascular conduit with excellent long-term prognosis is ITA, especially implanted to the left anterior descending artery (LAD) [5]. RA and particularly SV are considered as worse aorto-coronary grafts than ITA. Additionally, cardiac surgeons still debate which vessels should be used to bypass other coronary arteries $[6,7]$. It was proved that preoperative clinical and demographic status of patients undergoing CABG was also of importance with respect to conduits fate [8-10]. Moreover, the process of graft failure is multifactorial and thus many theories describing physiological properties of the aforementioned vessels have been proposed to explain such differences, including variability in expression of matrix metalloproteinases (MMP) together with their tissue inhibitors (TIMP), other cytokines as well as biologically active proteins within the vascular walls [11-13].

Many cell lines were considered to be involved in a phenomenon of graft failure [14-16]. More researchers stress a crucial role of macrophages in pathogenesis of aorto-coronary grafts failure $[17,18]$. It was previously found that distribution of macrophages, defined as CD68+ cells, differed between vessels with favorable and poor long-term outcomes [19]. The vascular segments with relatively high representation of macrophages in the inner vascular layers were found to be more prone to development of preterm graft disease related to early CAD symptoms recurrence.

The purpose of this study was to find any ultrastructural differences in CD68+ cells between routinely used aortocoronary bypass grafts.

\section{Material and Methods Patients}

This study involved 50 patients (37 male (74\%) and 13 women (26\%) with the mean age of $63.4 \pm 9.2$ years who underwent elective CABG procedures with the simultaneous application of RA and SV segments.

\section{Biological material obtaining}

During CABG procedures, surplus segments of vessels used as aorto-coronary grafts and at least one centimeter in length were taken for ultrastructural studies. The arterial grafts were harvested pedicled with the surrounding tissues to avoid any accidental microinjuries. All vessels were obtained in the standard manner applying a full skin incision over the entire course of them. Moreover, measures such as avoidance of touching ('no-touch method'), excessive manipulation and dilatation as well as high-energy electrocauthery were applied to minimize surgical trauma.

Vessels with macroscopic abnormalities such as signs of atherosclerosis (only in RA segments), inflammation, abnormally dilated or with iatrogenic injuries were not used both by surgeons as well as for ultrastructural examinations.

\section{Preparation for histological examinations}

Following macroscopic inspection harvested vascular segments were divided into two parts, one for analysis in transmission electron microscopy (TEM) were immersed in phosphate-buffered $2.5 \%$ glutaraldehyde $(0.05 \mathrm{M}, \mathrm{pH} 7.4)$ while the another one in freshly prepared Boiun solution for light microscopy. The further preparatory steps for microscopic examination were previously described in the details [18].

\section{Calculation of CD68-positive cells in light microscope study}

In immunohistochemical analysis the Dako REAL EnVision Detection System, Peroxidase/DAB, Rabbit/Mouse, K5007 (Dako, Copenhagen, Denmark) and mouse monoclonal anti-CD68 antibodies in dilution 1:100 (M0814, Dako) were used. Additionally, to identify macrophages in the vascular wall an eliminating assay with the following specific antibodies for lymphocyte subpopulations such as anti-CD20cy (1:400 dilution, M0755, Dako), anti-CD3 (1:50 dilution, M7254, Dako), anti-CD8 (1:100 dilution, M7103, Dako) and anti-CD30 (1:40 dilution, M0751, Dako) was carried out [18]. The peroxidase reaction was developed using diaminobenzidine.

Transverse vascular sections were analyzed under a brightfield microscope - Olympus BX 50 (OLYMPUS Optical Europe, Germany) equipped with a Mirax-Midi scanner (Carl Zeiss Microimaging $\mathrm{GmbH}$, Germany), coupled with a Panoramic Viewer, version 1.15.4, software (3DHISTECH Ltd., Budapest, Hungary). All of the analyses were performed blind using coded samples and included positive and negative controls. The negative controls consisted of sections incubated with nonimmune IgG1 (X0931, Dako) as well as sections in which the primary or secondary antibodies were omitted. In addition, serial sections were stained in subsequent experiments as positive controls and to determine 
the consistency of staining. The cells presenting expression of CD68 protein were counted first throughout the vessel wall then within the consecutive layers: the tunica intima, the tunica media and the tunica adventitia.

\section{Electron microscope examination}

For transmission electron microscopy (TEM), vascular samples were prepared in steps described previously [16]. Ultrathin sections were mounted on mesh nickel grids, counterstained with uranyl acetate then lead citrate and eventually examined using a JEM-1010 (JEOL, Tokyo, Japan) TEM. Typical utrastructural features of macrophages were assessed separately in all layer of the vascular wall and special attention was paid to cell membrane and cytoplasmic organella with respect to their biological activity.

\section{Data management and statistical analysis}

First, continuous variables were checked for normality with the use of the Shapiro-Wilk test. Those satisfying normal distribution criteria were presented as the means with standard deviations (sd). The remaining continuous data were expressed as medians with 25th to 75 th percentiles and then compared by means of the nonparametric Mann-Witney U test. A p value below 0.05 was considered as statistically significant. Analysis was performed with the use of statistical software Statistica 10.0 for Windows (StatSoft, Inc., Tulsa, OK, USA).

\section{Ethical approval}

The research related to human use has been complied with all the relevant national regulations, institutional policies and in accordance the tenets of the Helsinki Declaration, and has been approved by the authors' institutional review board or equivalent committee. The study protocol was approved by the Local Bioethical Committee (No 1201/08) and all patients expressed written consent to participate in this study.

\section{Results}

\section{A number of CD68+ cells}

Expression of CD68 protein was noted in all segments of the examined vascular segments although their number was in the wide range, in RA between 15 and 244 while 28 through 289 in SV. Median (25th; 75th percentile) of a total number of CD68+ cells, considered as macrophages, on the vascular transverse sections was smaller in RA (66 (43; 108)) than in SV $(95(67 ; 135))(\mathrm{p}<0.05)$ (Fig. 1).

\section{Distribution of macrophages}

Histological analysis revealed that not only the absolute number of CD68+ cells in the consecutive layers but also their percentage rate differed significantly between RA and SV. In the latter one, more macrophages were found in the inner strata of their vascular wall. In RA, markedly more CD68+ cells were found in the tunica adventitia. The rate of adventitial macrophages exceeded $70 \%$ in RA and ap-

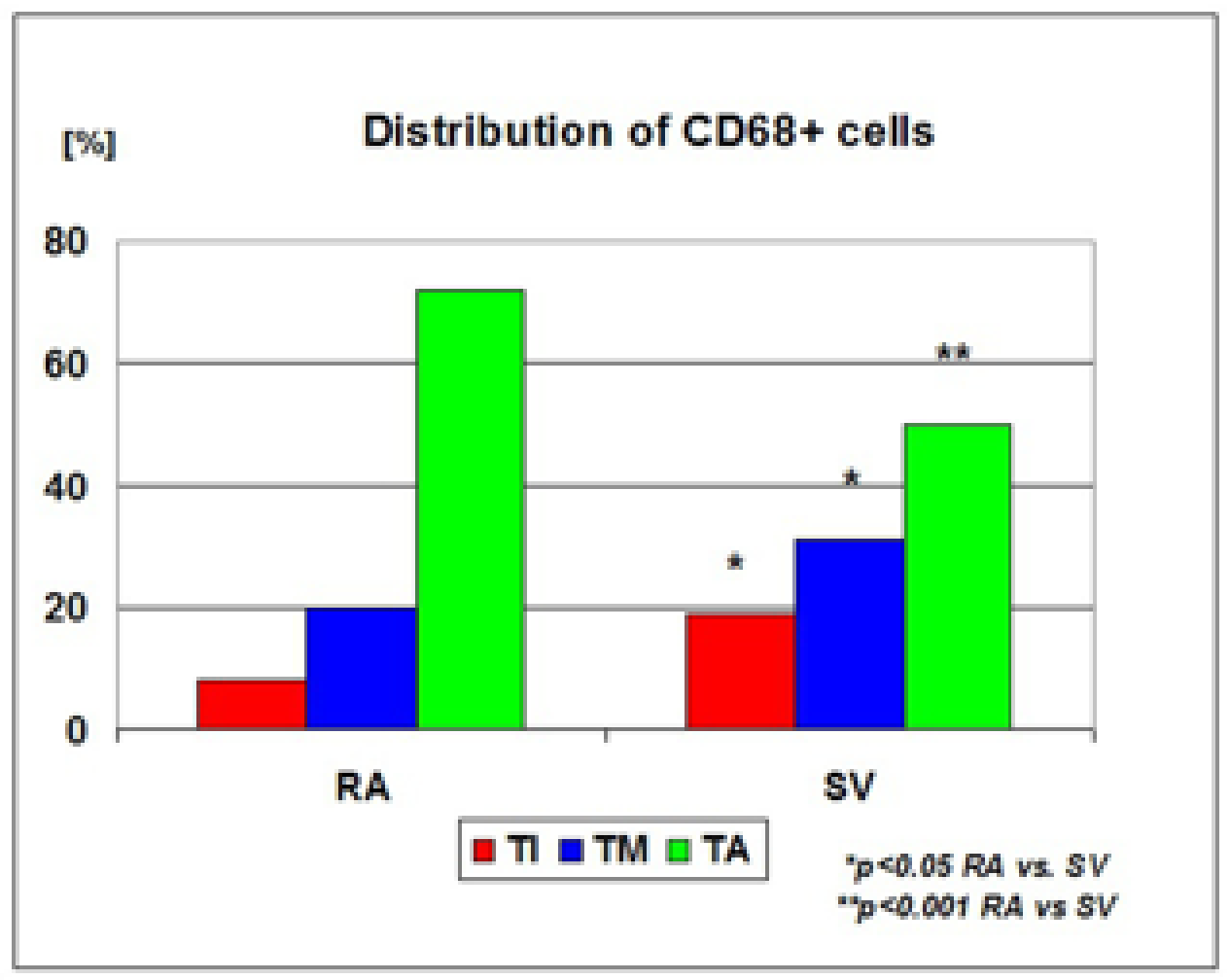

FIGURE 1 Distribution of CD68-positive cells on the vascular transverse sections

Abbreviations: $\mathrm{RA}=$ radial artery; $\mathrm{SV}=$ saphenous vein; $\mathrm{TA}=$ tunica adventitia; $\mathrm{TI}$ = tunica intima; $\mathrm{TM}=$ tunica media 
proximately $50 \%$ in the venous segments. The detailed data are outlined in table $\mathbf{1}$ (see also Fig. 1).

\section{Ultrastructure of CD68+ cells in TEM study Radial artery}

Detailed analysis of microelectrophotography of cells equipped with ultrastructures typical for macrophages revealed marked differences between these located in the tunica intima or media and in the tunica adventitia (Fig. 2).

Macrophages in the tunica intima and tunica media were very similar with respect to their morphology and manifested features of pronounced metabolic and phagocytic activity (Figs $\mathbf{2 a}$ and $\mathbf{2 b}$ ). Folded cellular membrane with irregular outline formed cytoplasmic protrusions with numerous caveolae and vesicles. Inside the cells, the cytoplasm was rich in organelles such as non-membraneous aggregation of electron-dense material and vesicles of varying electron density. Moreover, numerous lysosomes (both primary and mature) manifesting marked heterogeneity of size and shape as well as phagocytic vacuoles were also found. Centrally positioned large and euchromatic nuclei of irregular outline had one or two nucleoli inside.

Otherwise, the vast majority of macrophages in the tunica adventitia presented properties of unreactive residual cells filled with cytoplasm poor in cellular organelles with few heterogenous lysosomes and cisterns of rough endoplasmic reticulum, poorly developed Golgy apparatus and small mitochondria (Fig. 2c). Folded cellular membrane had only single caveolae-like protrusions. These cells were usually noted close to vasa vasorum and also in the layers of the tunica adventitia adjacent to the external elastic lamina.

\section{Saphenous vein}

In the subendothelial layer, macrophages were embedded between collagen fibers. Similarly to the arterial wall, the majority of cells detected as macrophages in the tunica intima and tunica media presented ultrastructure typical for biologically active cells such as numerous lysosomes, heterogenous phagocytic vesicles (Figs $\mathbf{3 a}$ and $\mathbf{3 b}$ ). These aforementio- ned findings were irrespective of the age of venous segments donors.

In the tunica adventitia there were macrophages among fibroblasts and smooth muscle cells. CD68+ cells were found predominantly in the areas close to vasa vasorum. Cytoplasmic equipment suggested deprived metabolic and phagocytic activity. Only single lysosomes were seen in the cytosol adjacent to the centrally positioned nuclei. In the majority of cells no caveolae and vesicles linked to the cellular membrane were detected (Fig. 3c).

\section{Discussion}

The findings of this study should be treated as complementary one for the previous articles focused on degeneration of aorto-coronary grafts and published by the same research team [13, 19, 20]. There it was pointed that macrophages may play a crucial role in grafts disease eventually leading to their occlusions and clinically recurrence of CAD symptoms [13]. Earlier conclusions were confined to statements about association between CD68+ cells distribution within vascular wall and good/ poor long-term outcomes [20]. Aorto-coronary conduits associated with poor angiographic and clinical outcomes usually had more macrophages in the tunica intima and tunica media. In the present analysis possible explanation of this phenomenon has been found. Different distribution of macrophages is likely related to the intrinsic ability of vascular wall to promote such processes as cellular transformations, migration and proliferation, neointima formation followed by atherosclerotic plaques development. In the inner layers, CD68+ cells are metabolically more active and thus probably better prepared for promotion of mechanisms involved in wall thickening and vessel lumen occlusion. Our study should be treated as support (on cellular level) of the previous theories that stressed an importance of macrophages in development of aorto-coronary conduits disease and eventual failure [21, 22]

However, it must be stressed that vascular segments obtained for this study were not applied as aorto-coronary grafts before. We can not exclude that surgical interposition of the vascular conduits

TABLE 1 An absolute number and percentage rate of CD68+ cells in the vascular layers

\begin{tabular}{|c|c|c|c|}
\hline \# & $\begin{array}{c}\text { RA } \\
{[N=50]}\end{array}$ & $\begin{array}{c}S V \\
{[N=50]}\end{array}$ & P VALUE \\
\hline Tunica intima & \begin{tabular}{|l|}
$3(2 ; 13)$ \\
$0.08(0.02 ; 0.12)$
\end{tabular} & \begin{tabular}{|l|}
$13(9 ; 23)$ \\
$0.19(0.14 ; 0.21)$
\end{tabular} & $\begin{array}{l}<0.001 \\
0.004\end{array}$ \\
\hline Tunica media & \begin{tabular}{|l|}
$12(6 ; 19)$ \\
$0.20(0.10 ; 0.24)$
\end{tabular} & \begin{tabular}{|l|}
$30(17 ; 43)$ \\
$0,31(0.23 ; 0.36)$
\end{tabular} & \begin{tabular}{|l|}
0.002 \\
0.002
\end{tabular} \\
\hline Tunica adventitia & \begin{tabular}{|l|}
$54(28 ; 90)$ \\
$0.72(0.60 ; 0.82)$
\end{tabular} & $\begin{array}{l}45(32 ; 74) \\
0.50(0.42 ; 0.58)\end{array}$ & $\begin{array}{l}0.015 \\
<0.001\end{array}$ \\
\hline
\end{tabular}

\# data are presented expressed as medians with percentiles (25th; 75th)

Abbreviations: $\mathrm{RA}=$ radial artery; $\mathrm{SV}=$ saphenous vein 

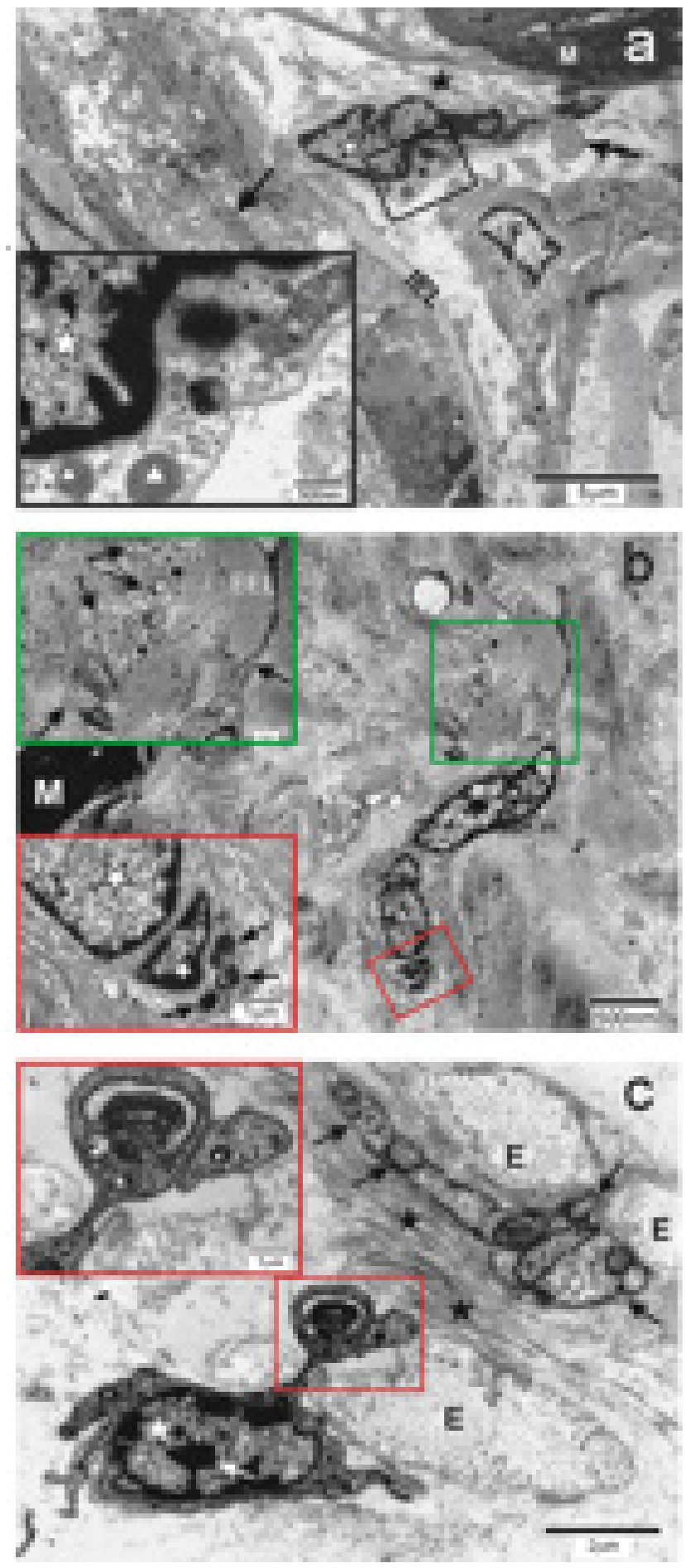

FIGURE 2 Transverse sections of the consecutive layers of radial artery

(a) In the subendothelial layer of RA wall, there are collagen fibers of connective tissue (black star), strata of elastic fibers of the internal elastic lamina (IEL) and a cell equipped with apparatus typical for macrophages adjacent to the latter one. A large, centrally located nucleus (white star) is surrounded by cytoplasm rich in polysomes and numerous vesicles. Lysosomes and phagosomes contain material of varying electron density (black frame - white triangles). Cellular podia of macrophages are found both in the tunica media (arrow) as well as in the tunica intima (arrow with double arrowhead). Two other cells, fibroblast (F) and vascular smooth muscle one (M) are seen in the close proximity. (b) Macrophage accompanied by smooth muscle cell (M) and embedded between collagen and elastic fibers of external elastic lamina (EEL) in the tunica media of RA is equipped with large nucleus (white star) and nucleolus inside (white arrowhead). Cell membrane with irregular outline creates many cytoplasmic podia (green frame - arrow with double arrowhead). There are also many vesicles and heterogenous lysosomes (red frame - arrows) within the cytoplasm. (c) Loosely organized collagen (black arrow) and elastic fibers (E) surround a macrophage with podia (arrow with double arrowhead). A large nucleus (white star) with nucleolus inside (white arrow) is in the central part of macrophage. A markedly reduced number of lysosomes is observed (red frame - white triangles) 

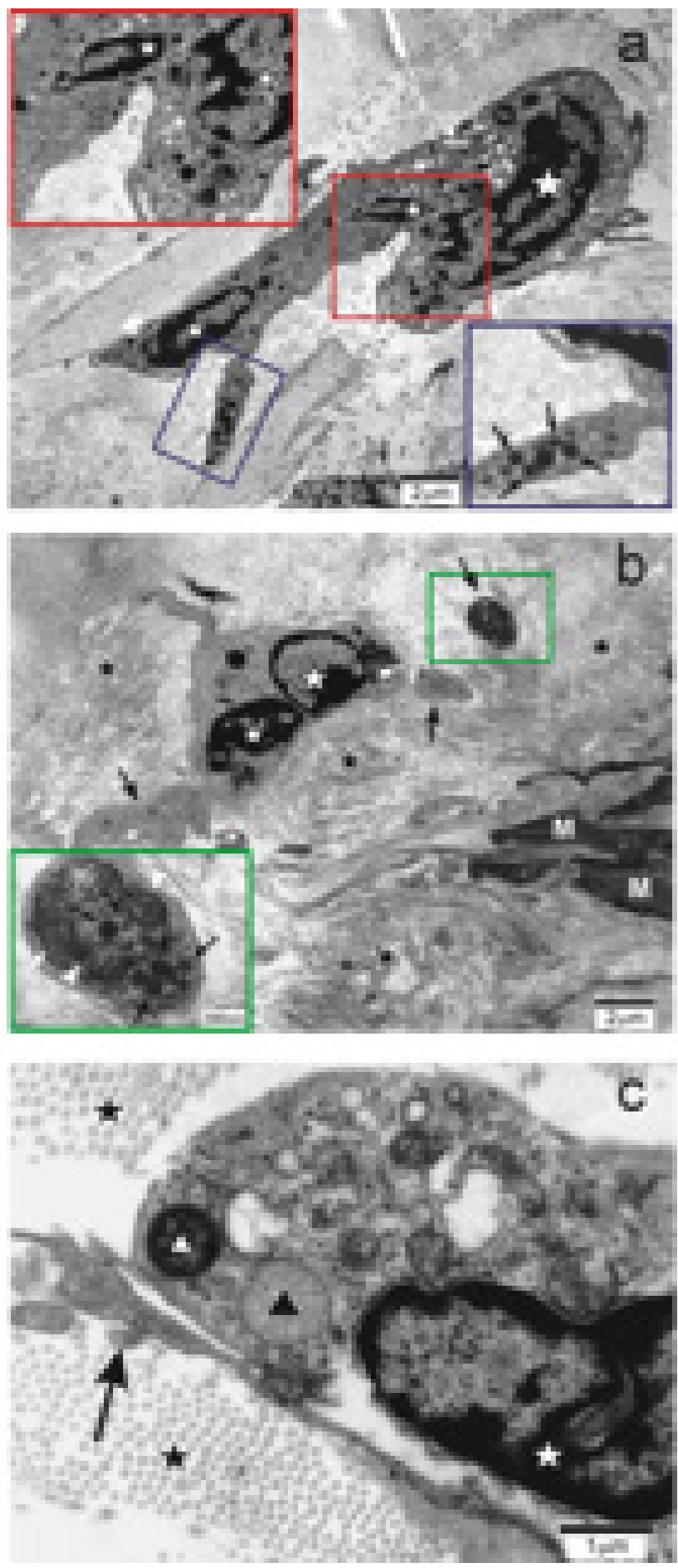

FIGURE 3 Microelectrophotography of macrophages in all layers of saphenous vein

(a) In the subendothelial layer of the tunica intima of SV, an "active" macrophage contains folded nucleus of irregular outline. In the closest proximity of its protrusions, vesicles of Golgi apparatus (arrows) and dilated cisterns of rough endoplasmic reticulum (arrows with double arrowhead) are seen. Numerous vesicles, phagosomes and dense granules of variable size (red frame) indicate on phagocytic cells activity. Dense cytoplasm rich in polysomes creates branched cilia with dense vesicles and halo zone typical for primary lysosome (violet frame - arrow). (b) Strongly folded cellular membrane (arrow) features a medial macrophage. Both primary (arrow with double arrowhead) and secondary (green frame - black arrows) lysosomes as well as mitochondria containing dense mitochondrial matrix (green frame - arrowhead) and cysterns of rough endothelial reticulum (green frame - white arrow) are within cytosol of podia. Nucleus (star) of irregular shape located in the central area is surrounded by cytoplasm rich in free ribosomes and polysomes. There are bundles of collagen fibers of irregular course in the extracellular matrix (black stars). (c) Heterochromatin is positioned peripherally in the nucleus. In its close proximity mutlivesicular body (black triangle) and lysosome are found. Cytoplasm is filled with size-and content-heterogenous vesicles. Cellular membrane creates pseudopodia (arrow) without any organelle inside. Collagen fibers filled extracellular matrix (black stars) 
into arterial circulation between ascending aorta and coronary arteries may change completely potential for transformation into more active cells. Maybe pronounced infiltration by cells with phagocytic activity is a marker of some processes (mechanical or metabolic microinjuries, infection or inflammation, thrombus formation) that had place before surgery and vessel harvesting. Although grafts were harvested with 'no-touch' technique and additional protective measures were applied, surgical microinjuries of harvested vascular segments were still possible.

Our finding may be an important voice in the discussion about the second best aorto-coronary graft after ITA. Even nowadays after publications of many clinical data, there is still no common consensus if RA or SV should be preferred $[23,24]$. Our findings may be treated as background for claiming that RA seems to be better aorto-coronary graft than SV because more active macrophages located in both the tunica intima and tunica media were found in the segments of the latter one. This study can help to support decision of cardiac surgeons regarding an optimal choice of vascular conduits. For sure, preoperative clinical status, coexisting diseases must be taken into account since some of them are associated with higher probability of vascular injury and remodeling, both involving macrophage activation [25-27].

The authors of this study are aware of limitations. First, this study is solely histological and ultrastructural analysis but the findings were not referred to the clinical outcomes. Due to complex study protocol and application of sophisticated scientific tools, a total number of transverse vascular sections had to be reduced to 50 in each group (RA and SV). Moreover, the examination were performed on the vessels before insertion them into aorto-coronary circulation as it was mentioned before. From ethical point of view it would not be possible to harvest such vessels from patients after a given period of follow-up. Application of experimental animal model, although valuable, would not be directly transferable to the human beings. At last, there are known drawbacks of immunohistochemical analysis of protein expression. However, two authors of this paper invited for research cooperation are histologists with great experience in the aforementioned method of protein expression analysis. Their expertise has been supported by numerous publications in the international and high-impacted peer-reviewed journals $[10,11,28,29]$.

\section{Conclusions}

Ultrastructural characteristics of both forms of macrophages infiltrating wall of aorto-coronary grafts is similar irrespective of the vessel type. More active cells in the inner layers of the venous conduits may contribute to their inferior outcomes compared to the arteries.

\section{Corresponding author}

Bartłomiej Perek MD, PhD, Department of Cardiac Surgery, 1/2 Długa St., 61-848 Poznan, Poland, phone: +48 618549 210, e-mail: bperek@ump.edu.pl.

\section{Acknowledgements}

This study was supported by the State Committee for Scientific Research (grant No. 5958/P01/2010/38).

\section{Conflict of interest statement}

The authors declare they have no conflict of interest.

\section{References}

1. Neumann FJ, Sousa-Uva M, Ahlsson A, Alfonso F, Banning AP, Benedetto $\mathrm{U}$ et al. $2018 \mathrm{ESC} / \mathrm{EACTS}$ Guidelines on myocardial revascularization. Kardiol Pol. 2018;76:1585-1664; DOI: 10.5603/KP.2018.0228.

2. Cuminetti G, Gelsomino S, Curello S, Lorusso R, Maessen JG, Hoorntje JC. Contemporary use of arterial and venous conduits in coronary artery bypass grafting: anatomical, functional and clinical aspects. Neth Heart J. 2017; 25: 4-13; DOI: 10.1007/s12471-016-0919-2.

3. Perek B, Misterski M, Stachowiak W, Buczkowski P, Stefaniak S, Puślecki $\mathrm{M}$ et al. The impact of coronary artery disease severity on late survival after combined aortic valve replacement and coronary artery bypass grafting - experience of a single cardiac surgery center. Kardiochir Torakochirurgia Pol. 2014;11:361-366. DOI: 10.5114/kitp.2014.47333.

4. Tugtekin SM, Alexiou K, Kappert U, Esche H, Joskowiak D, Knaut M et al. Coronary reoperation with and without cardiopulmonary bypass. Clin Res Cardiol. 2006;95:93-98; DOI: 10.1007/s00392-006-0335-5.

5. Lytle BW, Loop FD, Cosgrove DM, Ratliff NB, Easley K, Taylor PC. Longterm ( 5 to 12 years) serial studies of internal mammary artery and saphenous vein coronary artery bypass grafts. J Thorac Cardiovasc Surg. 1985;89:248-258

6. Zhang H, Wang ZW, Wu HB, Hu XP, Zhou Z, Xu P. Radial artery graft vs. saphenous vein graft for coronary artery bypass surgery: which conduit offers better efficacy? Herz 2014;39: 458-465; DOI: 10.1007/ s00059-013-3848-5

7. Taggart DP, Altman DG, Gray AM, Lees B, Gerry S, Benedetto U et al. Randomized Trial of Bilateral versus Single Internal-Thoracic-Artery Grafts. N Engl J Med. 2016;375:2540-2549. DOI: 10.1056/NEJMoa1610021.

8. Kieser TM, Curran HJ, Rose MS, Norris CM, Graham MM. Arterial grafts balance survival between incomplete and complete revascularization: a series of 1000 consecutive coronary artery bypass graft patients with 98\% arterial grafts. J Thorac Cardiovasc Surg. 2014;147:75-83; DOI: 10.1016/j.jtcvs.2013.08.003

9. Perek B, Malińska A, Nowicki M, Misterski M, Ostalska-Nowicka D, Jemielity M. Histological evaluation of age-related variations in saphenous vein grafts used for coronary artery bypass grafting. Arch Med Sci. 2012;8:1041-1047; DOI: 10.5114/aoms.2012.32412.

10. Perek B, Malinska A, Stefaniak S, Ostalska-Nowicka D, Misterski M, Zabel $\mathrm{M}$ et al. Predictive factors of late venous aortocoronary graft failure: ultrastructural studies. PLoS One. 2013;8:e70628; DOI: 10.1371/journal. pone.0070628.

11. Perek B, Malinska A, Misterski M, Ostalska-Nowicka D, Zabel M, Perek A et al. Preexisting high expression of matrix metalloproteinase-2 in tunica media of saphenous vein conduits is associated with unfavorable longterm outcomes after coronary artery bypass grafting. Biomed Res Int. 2013;2013:730721; DOI: 10.1155/2013/730721.

12. Perek B, Malińska A, Ostalska-Nowicka D, Puślecki M, Ligowski M, Misterski $\mathrm{M}$ et al. Cytokeratin 8 in venous grafts: a factor of unfavorable long-term prognosis in coronary artery bypass grafting patients. Cardiol J. 2013;20:583-591; DOI: 10.5603/CJ.2013.0142.

13. Perek B, Kowalska K, Nowicki M, Kempisty B, Puślecki M, Jankowski $\mathrm{M}$ et al. Variability in gelatinase expression in the walls of vessels used as aortocoronary conduits may impact long-term graft patency. Kardiol Pol. 2019;77:217-224; DOI: 10.5603/KP.a2019.0015.

14. Hassantash SA, Bikdeli B, Kalantarian S, Sadeghian M, Afshar H. Pathophysiology of aortocoronary saphenous vein bypass graft disease. Asian Cardiovasc Thorac Ann. 2008;16:331-336.

15. Hilker M, Tellmann G, Buerke M, Gloger K, Moersig W, Oelert H et al. Proliferative activity in stenotic human aortocoronary bypass grafts. Cardiovasc Pathol. 2002;11:284-290; DOI: 10.1016/s1054-8807(02)00113-8.

16. Schlitt A, Pruefer D, Buerke U, Russ M, Dahm M, Oelert H et al. Neutrophil adherence to activated saphenous vein and mammary endothelium after graft preparation. Ann Thorac Surg. 2006;81:1262-1268; DOI: 10.1016/j.athoracsur.2005.09.073.

17. Perek B, Kowalska K, Kempisty B, Nowicki A, Jankowski M, Nawrocki M et al. Role of macrophages in the pathogenesis of atherosclerosis and aortocoronary graft disease. J Biol Regul Homeost Agents. 2018;32:1055-1059. 
18. Malinska A, Perek B, Buczkowski P, Kowalska K, Ostalska-Nowicka D, Witkiewicz W et al. CD68 expression in aortocoronary saphenous vein bypass grafts. Histochem Cell Biol. 2013;140:183-188; DOI: 10.1007/ s00418-012-1069-2.

19. Perek B, Kowalska K, Kempisty B, Nawrocki M, Nowicki M, Puślecki $\mathrm{M}$ et al. Infiltration of $\mathrm{CD} 68+$ cells correlates positively with matrix metalloproteinase 2 expression in the arteries used as aortocoronary bypass grafts. Possible clinical implications. Cardiol J. 2019 Sep 6; DOI: 10.5603/ CJ.a2019.0087. [Epub ahead of print]

20. Perek B, Kowalska K, Kempisty B, Nowicki M, Dyszkiewicz-Konwińska M, Puślecki M, et al. Gender and age-related variability of macrophage representation in the internal thoracic artery wall: does it matter? J Biol Regul Homeost Agents. 2018 Jul-Aug;32(4):791-802.

21. Chung JH, Jeon HJ, Hong SY, Lee DL, Lee KH, Kim SH et al. Palmitate promotes the paracrine effects of macrophages on vascular smooth muscle cells: the role of bone morphogenetic proteins. PLoS One. 2012; 7: e29100; DOI: 10.1371/journal.pone.0029100.

22. Chatrou ML, Cleutjens JP, van der Vusse GJ, Roijers RB, Mutsaers PH, Schurgers LJ. Intra-Section analysis of human coronary arteries reveals a potential role for micro-calcifications in macrophage recruitment in the early stage of atherosclerosis. PLoS One. 2015;10:e0142335; DOI: 10.1371/journal.pone.0142335.

23. Zhu Y, Chen A, Wang Z, Liu J, Cai J, Zhou M, Zhao Q. Ten-year real-life effectiveness of coronary artery bypass using radial artery or great saphenous vein grafts in a single centre Chinese hospital. Interact Cardiovasc Thorac Surg. 2017;25:559-564; DOI: 10.1093/icvts/ivx174.

24. Petrovic I, Nezic D, Peric M, Milojevic P, Djokic O, Kosevic D et al. Radial artery vs saphenous vein graft used as the second conduit for surgical myocardial revascularization: long-term clinical follow-up. J Cardiothorac Surg. 2015;10:127; DOI: 10.1186/s13019-015-0331-9.

25. Qi D, Wei M, Jiao S, Song Y, Wang X, Xie G et al. Hypoxia inducible factor $1 \alpha$ in vascular smooth muscle cells promotes angiotensin II-induced vascular remodeling via activation of CCL7-mediated macrophage recruitment. Cell Death Dis. 2019;10:544; DOI: 10.1038/s41419-019-1757-0.

26. Harwani SC. Macrophages under pressure: the role of macrophage polarization in hypertension. Transl Res. 2018;191:45-63; DOI: 10.1016/j. trsl.2017.10.011.

27. Ahmed M, de Winther MPJ, Van den Bossche J. Epigenetic mechanisms of macrophage activation in type 2 diabetes. Immunobiology. 2017;222:937. 943; DOI: $10.1016 /$ j.imbio.2016.08.011.

28. Malinska A, Podemska Z, Perek B, Jemielity M, Buczkowski P, Grzymislawska $\mathrm{M}$ et al. Preoperative factors predicting saphenous vein graft occlusion in coronary artery bypass grafting: a multivariate analysis. Histochem Cell Biol. 2017;148:417-424; DOI: 10.1007/s00418-017-1574-4.

29. Nowicki M, Misterski M, Malinska A, Perek B, Ostalska-Nowicka D, Jemielity $\mathrm{M}$ et al. Endothelial integrity of radial artery grafts harvested by minimally invasive surgery--immunohistochemical studies of CD31 and endothelial nitric oxide synthase expressions: a randomized controlled trial. Eur J Cardiothorac Surg. 2011;39:471-477; DOI: 10.1016/j.ejcts.2010.08.005. 